\title{
Near Point Algorithm and Approximate Beam Method for Solving Variational Inclusion Problem
}

\author{
ZUO Ji-feng ${ }^{1, a}$, WANG Xue-chang ${ }^{2, b}$ \\ ${ }^{1}$ College of Science, School of Science Agricultural University of Hebei, Baoding 071001, China \\ ${ }^{2}$ Basic Course Department, Heibei College of Science and Technology, Baoding 071000, China \\ azjf79@sohu.com.cn, biolet_wxc@163.com
}

\begin{abstract}
Key words: near point algorithm; approximate beam method; variational inclusions; monotone operators
\end{abstract}

\begin{abstract}
It is well known that the variational inclusions are equivalent to the fixed point problems. A hybrid proximal point algorithm is considered for solving the general variational inclusions. And convergence analysis for this algorithm is explored along with some results on the resolvent operator corresponding to $(A, \eta)$-maximal operators.
\end{abstract}

\section{Introduction and preliminaries}

Variational inequalities and variational inclusions are among the most interesting and important mathematical problems and have been studied intensively in the past years, since they have wide applications in the optimization and control, economics and transportation equilibrium, engineering science. For these reasons, many existence results and iterative algorithms for various variational inclusions have been studied. For details, we refer the reader to [1-10].

Pennanen [11] over-relaxed the Eckstein-Bertsekas proximal point algorithm [12] and has shown that the sequence converges linearly to a solution to the following variational inclusion problem: for finding $x \in H$,such that $0 \in M(u)$, where $H$ is Hilbert space, $M: H \rightarrow 2^{H}$ is a set-valued mapping. On the basis of this new version of the proximal point algorithm, Pennanen [11] studied a localized version of the maximal monotonicity, and has shown that it ensures the local convergence of the over-relaxed proximal point algorithm. Furthermore, the local convergence of multiplier methods for a general class of problems is established. This, in a way, presents specializations as new convergence results for multiplier methods for nonmonotone variational inequalities and nonconvex nonlinear programming.

Recently Verma [13] develop a hybrid version of the Eckstein-Bertsekas proximal point algorithm based on the notions of $A$-maximal monotonicity [4] and $(A, \eta)$-maximal monotonicity [14] for solving the variational inclusion problem (1). These notions generalize the general class of maximal monotone set-valued mappings, including the notion of $H$-maximal monotonicity introduced by Fang and Huang [15] in a Hilbert space.

Motivated and inspired by the research work going on this field, in this paper, we extend the hybrid proximal point algorithm to solve the general variational inclusion.

Let $H$ be a real Hilbert space with the norm $\|$.$\| and the inner product \langle.,$.$\rangle For given nonlinear$ operators $T, g: H \rightarrow H$ and an $(A, \eta)$-maximal monotone mapping $\mathrm{M}: \mathrm{H} \rightarrow 2^{H}$, we consider the problem of finding $u \in H$ such that

$$
0 \in T u+M(g(u))
$$

which is called the general variational inclusion. Variational inclusions have been studied and considered by many authors.

For $g=I$, the identity operator, problem (2) is equivalent to finding $\mathrm{u} \in \mathrm{H}$ such that

$$
0 \in T u+M(u)
$$

which is known as the variational inclusion problem.

If $\mathrm{M}()=.\partial \varphi($.$) , where \varphi($.$) is the subdifferential of a proper, convex and semi-continuous$ function $\varphi: H \rightarrow R \cup\{+\infty\}$, then problem (2) reduces to finding $u \in H$ such that 


$$
0 \in T(u)+\partial \varphi(g(u))
$$

or equivalently, finding $u \in H, g(u) \in H$, such that

$$
\langle T u, g(v)-g(u)\rangle+\varphi(g(v))-\varphi(g(u)) \geq 0, \forall g(v) \in H
$$

Definition 1.1 Let $M: H \rightarrow 2^{H}$ be a multivalued mapping, and $F: H \rightarrow H$ be another operator. The map $M$ is said to be :

(i)(r)-strongly monotone if there exists a positive constant $r$ such that: $\forall u, v \in H, u^{*} \in M(u), v^{*} \in$ $M(v)$

$$
\left\langle u^{*}-v^{*}, u-v\right\rangle \geq r\|u-v\|^{2}
$$

(ii)(m)-relaxed monotone if there exists a positive constant $m$ such that: $\forall u, v \in H, u^{*} \in M(u), v^{*} \in$ $M(v)$

$$
\left\langle u^{*}-v^{*}, u-v\right\rangle \geq(-m)\|u-v\|^{2}
$$

(iii)(r)-strongly monotone with respect to $F$ if there exists a positive constant $r$ such that: $\forall u, v \in$ $H, u^{*} \in M(u), v^{*} \in M(v)$

$$
\left\langle u^{*}-v^{*}, F u-F v\right\rangle \geq r\|u-v\|^{2}
$$

Definition 1.2 Let $M: H \rightarrow 2^{H}$ be a multivalued mapping on $H$, and let $\eta: H \times H \rightarrow H$ be another mapping. The map $M$ is said to be $(r, \eta)$-strongly monotone if there exists a positive constant $r$ such that $\forall u, v \in H, u^{*} \in M(u), v^{*} \in M(v)$

$$
\left\langle u^{*}-v^{*}, \eta(u, v)\right\rangle \geq r\|u-v\|^{2}
$$

Where $\eta$ is said to be $(\tau)$-Lipschitz continuous if there is a positive constant $\tau$ such that

$$
\eta\|(u, v)\| \leq \tau\|u-v\| .
$$

Definition 1.3 ([4]) Let $A: H \rightarrow H$ be $(r)$-strongly monotone. The map $M \rightarrow 2^{H}$ is said to be A-maximal monotone if

(i) $M$ is $(m)$-relaxed monotone,

(ii) $R(A+\rho M)=H$, for $\rho>0$

Definition 1.4 Let $A: H \rightarrow H$ be $(r, \eta)$-strongly monotone. The map $M: H \rightarrow 2^{H}$ is said to be $(A, \eta)$-maximal monotone if

(i) $M$ is $(m, \eta)$-relaxed monotone,

(ii) $R(A+\rho M)=H$ for $\rho>0$

Definition 1.5 Let $A: H \rightarrow H$,be an $(r, \eta)$-strongly monotone mapping and let $M: H \rightarrow 2^{H}$ be an $(A, \eta)$-maximal monotone mapping. Then the generalized resolvent operator $J_{\rho, A}^{M, \eta}: H \rightarrow H$ is defined by

$$
J_{\rho, A}^{M, \eta}(u)=(A+\rho M)^{-1}(u)
$$

Proposition 1.1 ([14]). Let $A$ : $H \rightarrow H$ be an $(r, \eta)$-strongly monotone single-valued mapping and let $M: H \rightarrow 2^{H}$ be an $(A, \eta)$-maximal monotone mapping. Then $A+\rho M$ is maximal monotone for $\rho>0$.

Proposition 1.2 Let $A$ : $H \rightarrow H$ be an $(r, \eta)$-strongly monotone mapping and let $M: H \rightarrow 2^{H}$ be an $(A, \eta)$-monotone mapping. Then the operator $(A+\rho M)^{-1}$ is single-valued.

\section{Hybrid proximal point algorithms}

This section deals with a hybrid proximal point algorithm for the over-relaxed version of the Eckstein-Bertsekas proximal point algorithm [12] and its application to approximation solvability of the inclusion problem (2) based on the $(A, \eta)$-maximal monotonicity.

Lemma 2.1 ([14]) Let $H$ be a real Hilbert space. Let $A: H \rightarrow H$ be an $(r, \eta)$-strongly monotone, $M: H \rightarrow 2^{H}$ be an $(A, \eta)$-maximal monotone, and $\eta: H \times H \rightarrow H$ be $(\tau)$-Lipschitz continuous. Then the generalized resolvent operator associated with $M$ and defined by

$$
J_{\rho, A}^{M, \eta}(u)=(A+\rho M)^{-1}(u), \forall u \in H,
$$

is $\left(\frac{\tau}{r-\rho m}\right)$-Lipschitz continuous. Furthermore, we have

$\left\langle J_{\rho, A}^{M, \eta}(u)-J_{\rho, A}^{M, \eta}(v), u-v\right\rangle \leq \frac{\tau}{r-\rho m}\|u-v\|^{2}$.

Theorem 2.1 Let $H$ be a real Hilbert space. Let $A: H \rightarrow 2^{H}$ be $(r, \eta)$-strongly monotone, $M: H \rightarrow$ 
$2^{H}$ be $(A, \eta)$-maximal monotone. Then the following statements are mutually equivalent:

(i) $u \in H$ is a solution to (2);

(ii) $u \in H$ we have

$g(u)=J_{\rho, A}^{M, \eta}(A(g(u))-\rho T(u))$,

where $J_{\rho, A}^{M, \eta}(u)=(A+\rho M)^{-1}(u)$ and $0<\rho<\frac{r}{m}$

In the following theorem, we apply the hybrid proximal point algorithm to approximate the solution of (1), and as a result, we end up showing linear convergence.

Theorem 2.2 Let $H$ be a real Hilbert space, let $\eta: H \times H$ be a single-valued operator and a Lipschitz continuous operator with constant $\tau, \eta: H \times H$ be a strongly $\eta$-monotone, Lipschitz continuous operator with constants $r$, s respectively, $g: H \rightarrow H$,be strongly monotone and Lipschitz continuous with constants $\gamma$ and $t$ respectively. Let $T: H \rightarrow H$ be $r_{1}$ strongly monotone with respect to $A^{\circ} g$ and $s_{1}$-Lipschitz continuous. Let $M: H \rightarrow 2^{H}$ be $(A, \eta)$-maximal monotone. For an arbitrarily chosen initial point $x_{0}$, suppose that the sequence $\left\{x_{n}\right\}$ is generated by the following iterative procedure

and $y_{n}$ satisfies

$$
x_{n+1}=\left(1-\alpha_{n}\right) x_{n}+\alpha_{n} y_{n}, \forall n \geq 0,
$$

$$
\left\|y_{n}-g^{-1} J_{\rho_{n}, A}^{M, \eta}\left(A g\left(x_{n}\right)-\rho_{n} T\left(x_{n}\right)\right)\right\| \leq \delta_{n}\left\|y_{n}-x_{n}\right\|,
$$

where $J_{\rho_{n}, A}^{M, \eta}=\left(A+\rho_{n} M\right)^{-1}$ and $\left\{\delta_{n}\right\},\left\{\alpha_{n}\right\},\left\{\rho_{n}\right\} \subset(0,+\infty)$ are scalar sequences such that $\sum_{n=0}^{\infty} \delta_{0}<\infty, \delta_{n} \rightarrow 0, \alpha=\lim _{n \rightarrow \infty} \sup \alpha_{n}, \rho_{n} \uparrow \rho \leq \infty, \alpha_{n}<1$.

Then the sequence $\left\{x_{n}\right\}$ converges linearly to a solution of (2) with convergence rate

$$
\sqrt{1-2 \alpha\left[1-\frac{1}{2} \alpha-(1-\alpha) v \mu-\frac{1}{2} \alpha \frac{1}{\gamma^{2}} v^{2} \mu^{2}\right]}<1
$$

Where $v=\frac{s \tau}{r-\rho m}, \mu=\sqrt{s^{2} t^{2}-2 \rho r_{1}+\rho^{2} s_{1}^{2}}$

for $c=r-\rho m$,

$$
\begin{gathered}
c<\frac{(1-\alpha) s-\sqrt{((1-\alpha) s)^{2}+(2-\alpha) \alpha \frac{1}{\gamma^{2}} s^{2} \tau^{2} \mu^{2}}}{2-\alpha} \\
(1-\alpha) s>\sqrt{((1-\alpha) s)^{2}+(2-\alpha) \alpha \frac{1}{\gamma^{2}} s^{2} \tau^{2} \mu^{2}}
\end{gathered}
$$

and

$c>\frac{(1-\alpha) s-\sqrt{((1-\alpha) s)^{2}+(2-\alpha) \alpha \frac{1}{\gamma^{2}} s^{2} \tau^{2} \mu^{2}}}{2-\alpha}$

Proof: By the strong monotonicity of $g$ with constant $\gamma$

$\|g(u)-g(v)\|\|u-u\| \geq\langle g(u)-g(v), u-v\rangle \geq \gamma\|u-v\|^{2}$

That is $\|g(u)-g(v)\| \geq \gamma\|u-v\|$ thus, the above formula implies that

$$
\left\|g^{-1}(u)-g^{-1}(v)\right\| \leq \frac{1}{\gamma}\|u-v\|
$$

$g^{-1}$ is single-valued operator and

Let $x^{*}$ be a solution of the problem (2). It follows from Theorem 2.1 that

$$
\mathrm{g}\left(x^{*}\right)=J_{\rho_{n}, A}^{M, \eta}\left(\operatorname{Ag}\left(x^{*}\right)-\rho_{n} T\left(x^{*}\right)\right) .
$$

For all $n \geq 0$, we write

$$
z_{n+1}=\left(1-\alpha_{n}\right) x_{n}+\alpha_{n} g^{-1} J_{\rho_{n}, A}^{M, \eta}\left(A g\left(x_{n}\right)-\rho_{n} T\left(x_{n}\right)\right)
$$

We have

$$
\begin{aligned}
& \left\|z_{n+1}-x^{*}\right\|^{2} \leq\left(1-\alpha_{n}\right)^{2}\left\|x_{n}-x^{*}\right\|^{2} \\
& +2 \alpha_{n}\left(1-\alpha_{n}\right) \frac{v_{n}}{\gamma}\left\|x_{n}-x^{*}\right\| \times\left\|A g\left(x_{n}\right)-\rho_{n} T\left(x_{n}\right)-A g\left(x^{*}\right)+\rho_{n} T\left(x^{*}\right)\right\| \\
& +\frac{v_{n}^{2}}{\gamma^{2}}\left\|A g\left(x_{n}\right)-\rho_{n} T\left(x_{n}\right)-A g\left(x^{*}\right)+\rho_{n} T\left(x^{*}\right)\right\|^{2},
\end{aligned}
$$


where $v_{n}=\frac{s \tau}{r-\rho_{n} m}$

By the assumptions, we have

$\left\|A g\left(x_{n}\right)-\rho_{n} T\left(x_{n}\right)-A g\left(x^{*}\right)+\rho_{n} T\left(x^{*}\right)\right\|^{2}$

$=\left\|A g\left(x_{n}\right)-A g\left(x^{*}\right)\right\|^{2}-2 \rho_{n}\left\langle T\left(x_{n}\right)-T\left(x^{*}\right), A g\left(x_{n}\right)-A g\left(x^{*}\right)\right\rangle+\rho_{n}^{2}\left\|T x_{n}-T x^{*}\right\|$

$\leq\left(s^{2} t^{2}-2 \rho_{n} r_{1}+\rho_{n}^{2} s_{1}^{2}\right)\left\|x_{n}-x^{*}\right\|^{2}$

$=\mu_{n}^{2}\left\|x_{n}-x^{*}\right\|^{2}$

where $\mu_{n}=\sqrt{s^{2} t^{2}-2 \rho_{n} r_{1}+\rho_{n}^{2} s_{1}^{2}}$

It follows that:

$\left\|z_{n+1}-x^{*}\right\|^{2}$

$\leq\left\{1-2 \alpha_{n}\left[1-\frac{\alpha_{n}}{2}-\left(1-\alpha_{n}\right) v_{n} \mu_{n}-\frac{\alpha_{n} v_{n}^{2} \mu_{n}^{2}}{2 \gamma^{2}}\right]\right\} \times\left\|x_{n}-x^{*}\right\|^{2}$

$=\theta_{n}^{2}\left\|x_{n}-x^{*}\right\|^{2}$

Where

$\theta_{n}=\sqrt{1-2 \alpha_{n}\left[1-\frac{\alpha_{n}}{2}-\left(1-\alpha_{n}\right) v_{n} \mu_{n}-\frac{\alpha_{n} v_{n}^{2} \mu_{n}^{2}}{2 \gamma^{2}}\right]}$

Since $x_{n+1}=\left(1-\alpha_{n}\right) x_{n}+\alpha_{n} y_{n}$, we have $x_{n+1}-x_{n}=\alpha_{n}\left(y_{n}-x_{n}\right)$

It follows that:

$\left\|x_{n+1}-z_{n+1}\right\|=\left\|\alpha_{n}\left(y_{n}-g^{-1} J_{\rho_{n}, A}^{M, \eta}\left(A g\left(x_{n}\right)-\rho_{n} T\left(x_{n}\right)\right)\right)\right\| \leq \alpha_{n} \delta_{n}\left\|y_{n}-x_{n}\right\|$

Next we have:

$\left\|x_{n+1}-x^{*}\right\| \leq\left\|z_{n+1}-x_{n+1}\right\|+\left\|z_{n+1}-x^{*}\right\| \leq\left\|z_{n+1}-x^{*}\right\|+\alpha_{n} \delta_{n}\left\|y_{n}-x_{n}\right\|$

$=\left\|z_{n+1}-x^{*}\right\|+\delta_{n}\left\|x_{n+1}-x_{n}\right\| \leq\left\|z_{n+1}-x^{*}\right\|+\delta_{n}\left\|x_{n+1}-x^{*}\right\|+\delta_{n}\left\|x_{n}-x^{*}\right\|$

This implies that:

$$
\left\|x_{n+1}-x^{*}\right\| \leq \frac{\theta_{n}+\delta_{n}}{1-\delta_{n}}\left\|x_{n}-x^{*}\right\|
$$

where

$\lim \sup \frac{\theta_{n}+\delta_{n}}{1-\delta_{n}}=\lim \sup \theta_{n}$

$=\sqrt{1-2 \alpha\left[1-\frac{1}{2} \alpha-(1-\alpha) v \mu-\frac{1}{2} \alpha \frac{1}{\gamma^{2}} v^{2} \mu^{2}\right]}<1$

where $v=\frac{s \tau}{r-\rho m}, \mu=\sqrt{s^{2} t^{2}-2 \rho r_{1}+\rho^{2} s_{1}^{2}}$

\section{Conclusion}

In this paper, we develop the hybrid point algorithm for solving the general variational inclusions and obtain the linearly convergence of the hybrid proximal point algorithm based on $(A, \eta)$-monotonicity. Our result extends the results of J. Eckstein [12] and R. Verma [13].

\section{Acknowledgments}

This work is supported by the Project of Baoding science and Technology Bureau (No.13zs002), Key Project of Chinese Ministry of Education (No.207014) and the Natural Science Foundation of HeBei University (No.2006Q04).

\section{References}

[1] Z. Qi, Y. Tian, S. Yong, Laplacian twin support vector machine for semi-supervised classification, Neural Networks 35 (2012) 46-53.

[2] Y. Shao, N. Deng, Z. Yang, W. Chen, Z. Wang, Probabilistic outputs for twin support vector machines, Knowledge-Based Systems 33 (2012) 145-151. 
[3] Z. Qi, Y. Tian, S. Yong, Twin support vector machine with universum data, Neural Networks 36C (2012) 112-119. doi:doi: 10.1016/j.neunet.2012.09.004.

[4] J. W. Peng, On a new system of generalized mixed quasi-variational-like inclusions with $(\mathrm{H}$, $\eta$ )- accretive operators in real q-uniformly Banach spaces, Nonliner Anal. (2007) doi:10.1016/j.na.2006.11.054.

[5] H. Y. Lan, Y. J. Cho, R. U. Verma, Nonlinear relaxed cocoercive variational inclusions involving (A, $\eta$ )-accretive mappings in Banach spaces, Comput. Math. Appl. 51 (2006) 1529-1538.

[6] T. Pennanen, Local convergence of the proximal point algorithm and multiplier methods without monotonocity, Math. Oper. Res. 27(1) (2002)170-191.

[7] J. Eckstein, D.P. Bertsekas, On the DouglasCRachford splitting method and the proximal point algorithm for maximal monotone operators, Math. Program. 55(1992) 293-318.

[8] R. U. Verma, A hyrid proximal point algorithm based on the $(A, \eta)$-maximal monotonocity framework, Appl. Math. Lett. 21(2008) 142-147.

[9] R.U. Verma, Sensitivity analysis for generalized strongly monotone variational inclusions based on the $(A, \eta)$-resolvent operator technique, Appl. Math. Lett. 19 (2006) 1409C1413.

[10]Y.P. Fang, N.J. Huang, $H$-monotone operators and system of variational inclusions, J. Math. Anal. Appl. 327(1)(2007) 481-493.

[11]N.J. Huang, A new completely general class of variational inclusions with noncompact valued mapping, Comput. Math. Appl. 35(10)(1998). 